\title{
EFFECT OF WEATHERING ON DYNAMIC MECHANICAL PROPERTIES OF THE MULTILAYER POLYAMIDE//ETHYLENE VINYL ALCOHOL COPOLYMER//POLYAMIDE//POLYETHYLENE FILM
}

\begin{abstract}
Summary
Commercial co-extruded multilayer film of $25 \mu \mathrm{m}$ thickness was tested for use as an airborne module. The module in a form of a cylindrical balloon rotates at high altitudes in order to convert wind energy to electrical energy. The film consists of low-density polyethylene, ethylene vinyl alcohol copolymer, two layers of polyamide 6 (PA6//EVOH//PA6//LDPE) and tie layers between them.

The asymmetric film was aged in a weathering chamber at room temperature, with either the PA6 surface or the LDPE surface directly exposed to the xenon-arc light. The exposure of 1000 hours to a rainy and a sunny cycle ruined the film; therefore, the film was exposed to shorter sunny cycles only, for 3, 6, 16, 44, and 67 hours. The effect of solar radiation on tensile properties was tested in the machining and transverse directions by means of tensile testing at room temperature and dynamic mechanical analysis (DMA) at temperatures ranging from 83 to $373 \mathrm{~K}$. In order to get a better insight into structural changes, the Fourier transform infrared spectroscopy (FTIR) analysis is performed. Water vapour sorption and the melting temperature range of the unexposed film were determined by means of thermogravimetric analysis (TGA) and optical microscopy, respectively.

The tensile tests indicate a high anisotropy of the coextruded film. After irradiation, there is an increase in crystallinity degree. Kinetics of crystallization and hence the mechanical properties depend on the exposed surface.
\end{abstract}

Keywords: $\quad$ Multilayer film; Weathering; Mechanical properties; Viscoelasticity; FTIR spectroscopy

\section{Introduction}

Lightweight design is in growing demand. The main reason for that is a reduction in energy consumption, but it is also the functionality of certain structures that requires a mass reduction. An example for the latter is a rotating balloon that is a part of a structure for future technologies for renewable energy sources based on wind energy. The cylindrical balloons 
used as buoyant objects are filled with a light gas, such as hydrogen, helium or nitrogen, and rotate at altitudes between 0 and $2000 \mathrm{~m}$ passing through big temperature changes. Besides the demands for acceptable mechanical, barrier properties and low density, there are particular demands on such a product due to the fact that the outdoor use includes a large temperature range, especially if it is intended for higher altitudes. The exposure to the outdoor conditions requires resistance to solar radiation, mostly in the ultraviolet (UV) spectrum, and to moisture. The actual temperature to which objects that are used at altitudes from zero to several thousands of meters are exposed changes significantly. In addition, the geographical location and the season have an influence on the humidity, temperature, and UV intensity. Due to their low density compared to other structural materials, polymeric materials are often a good choice for lightweight structures, but they are often sensitive to UV light, moisture, biological agents, and pollutants. Furthermore, a great deal of their properties does not only depend on the degradation due to the UV exposure or the plasticizing effect of moisture, but they also greatly depend on temperature, especially those near the glass-transition.

The co-extruded multilayer polymer film Heptax (Gunze) was considered as a possible choice. The film is asymmetric as it has polyamide (PA) and polyethylene (PE) as outer layers plus ethylene vinyl alcohol polymer $(\mathrm{EVOH})$ layer and an additional layer of PA as inner layers. EVOH has good barrier properties, which prevent gas migrations in the balloon and out of it [1]. Compared to other polymers, EVOH has very good UV stability. PA 6 is an aliphatic polyamide which should be stable to wavelengths above $300 \mathrm{~nm}$; however chromophore groups formed during manufacturing absorb UV light at $320 \mathrm{~nm}$ and form radicals, which leads to degradation [2]. It is common knowledge that PE is a polymer which is sensitive to UV degradation. Both $\mathrm{EVOH}$ and $\mathrm{PA}$ have good mechanical properties, with a slight advantage of the latter. Low-density polyethylene (LDPE) and PA are outer layers because LDPE allows thermal bonding and PA allows printing on its surface. The sensitivity to moisture of EVOH and PA is very well described in literature and this effect has a strong influence on the material response to mechanical stresses. [3,4]

There were two main goals of this study: one was to test the effect of weathering on the structure and properties of the multilayer film and the other was to test whether there were any differences in the behaviour of the material with respect to the way the film was oriented (the machining or the transverse direction) and to the surface (PA or PE) facing outwards.

From the tensile test results it is clear that co-extrusion causes the anisotropy of the film. The weathering cycles influence the structure, which leads to an increase in the crystallinity degree and this, in turn, affects the mechanical properties and water sorption. Although the film is only $25 \mu \mathrm{m}$ thick and transparent, the kinetics of crystallization depends on the surface (PA or PE) directly exposed to irradiation.

\section{Experiment}

\subsection{Materials}

The multilayer polymer film under the commercial name Heptax (purchased by Gunze Limited) consists of seven biaxially-oriented co-extruded layers of five different compositions, four of them being polymer layers and three tie-layers between them. The film thickness is $25 \mu \mathrm{m}$ and its composition is polyamide (PA 6)// ethylene vinyl alcohol (EVOH)// polyamide (PA 6)// low-density polyethylene (LDPE). The film was co-extruded using a T-die. Heptax resists stretching and this property makes it appropriate for applications with pressure differences. Its specific weight is $26.23 \mathrm{~g} / \mathrm{m}^{2}$ and the density is $0.953 \mathrm{~g} / \mathrm{cm}^{3}$. 


\subsection{Weathering}

Samples were aged using a Suntest XLS+ (ATLAS), equipped with a xenon-arc lamp, simulating solar radiation. The irradiance on the surface of the tested samples was of wavelengths between $300 \mathrm{~nm}$ and $800 \mathrm{~nm}$, covering both the UV and the visible light, while the light intensity was $550 \mathrm{~W} / \mathrm{m}^{2}$. The first tests performed for 1000 hours with rainy and sunny periods according to EN ISO 4892-2 resulted in the disintegration of the film. In order to decouple the effects of sunny periods from those of rainy ones, the same material was exposed only to the sunny periods for shorter irradiation times of $3,8,16,44$, and 67 hours, with doses of $5869 \mathrm{~kJ} / \mathrm{m}^{2}, 15791 \mathrm{~kJ} / \mathrm{m}^{2}, 31630 \mathrm{~kJ} / \mathrm{m}^{2}, 86982 \mathrm{~kJ} / \mathrm{m}^{2}$, and $132450 \mathrm{~kJ} / \mathrm{m}^{2}$, without rainy cycles. The temperature of the chamber was maintained at $308 \mathrm{~K}$ and that of the reference black body (black standard) at $338 \mathrm{~K}$.

\subsection{Techniques}

\subsubsection{Tensile tests}

Co-extrusion of films and successive drawing resulted in anisotropy [5]. This was confirmed by the tensile strength testing according to EN ISO 527-3. The tensile tests of films and fabrics were performed on a test machine, manufactured by VEB Thüringer Industriewerk (DE), with the accuracy class 1.0 in the measurement range 0-950 N. Sample preparation included the cutting out of the film in a rectangular shape $15 \times 150 \mathrm{~mm}$ and the checking of all edges by a magnifying glass in order to avoid a stress concentration due to irregularities. The samples were placed in the tensile test machine with a distance between grips of $100 \mathrm{~mm}$. The test speed was $50 \mathrm{~mm} / \mathrm{min}$. Both the machining and transverse directions were tested.

\subsubsection{Dynamic mechanical analysis (DMA)}

DMA measurements were carried out on a Triton Technology DMA instrument using tension clamps for rectangular samples. The length of the samples was $20 \mathrm{~mm}$ and the width was $3 \mathrm{~mm}$. The span between the clamps was $7.5 \mathrm{~mm}$. Experiments were performed at a frequency of $1 \mathrm{~Hz}$ and at temperatures ranging from 83 to $373 \mathrm{~K}$. A frequency of $1 \mathrm{~Hz}$ was chosen for the DMA according to the majority of available publications, but the tests performed on thin films showed smoother curves at frequencies of 10 or $100 \mathrm{~Hz}$. Temperatures of $83 \mathrm{~K}$ were reached by means of liquid nitrogen at a cooling rate of $10 \mathrm{~K} / \mathrm{min}$. Measurements were performed in the heating cycle, at a rate of $2 \mathrm{~K} / \mathrm{min}$. A sinusoidal displacement was applied with amplitudes ranging from 16 to $18 \mu \mathrm{m}$. Due to the confirmed anisotropy, DMA was performed both in the machining and the transverse direction.

\subsubsection{Fourier transform infrared (FTIR) spectra}

FTIR spectra of the materials were recorded on a Bruker Vertex 70 FTIR spectrometer in the attenuated total reflectance mode. The spectra were obtained using a resolution of $1 \mathrm{~cm}^{-}$ 1 , averaged over 24 scans between 400 and $4000 \mathrm{~cm}^{-1}$. All films were characterized from both sides - the one directly exposed to the radiation, and the opposite one.

\subsubsection{Water vapour sorption}

The water vapour sorption of Heptax was examined by means of a thermal gravimetric analysis (TGA Q5000, TA Instruments). The cycle included drying at $0 \%$ of relative humidity followed by absorption at $333 \mathrm{~K}$ and $60 \%$ of relative humidity, and then completed by desorption. The temperature during drying increased from 298 to $333 \mathrm{~K}$ in about 35 minutes and after that it was kept constant. The mass of the $25 \mu \mathrm{m}$ thin sample was $5.871 \mathrm{mg}$. 


\subsubsection{Melting temperature ranges}

The melting temperature ranges of each layer were analysed by means of an optical microscope with a heating plate instead of a stage and with a thermometer included (Nagema). The temperature rate was controlled by means of a resistor which was set to 4 ${ }^{\circ} \mathrm{C} / \mathrm{min}$. The films were placed between two microscope slides and observed during heating.

\section{Results and discussion}

In order to test the stability of the film it was first conditioned for 1000 hours in the Xenon exposure system with rainy and sunny periods according to EN ISO 4892-2. After the long weathering, the film disintegrated and was not appropriate for mechanical tests. Therefore, the kinetics of the degradation was analysed for shorter sunny cycles.

\subsection{Tensile test results}

Tensile tests proved a high anisotropy related to the direction of the force applied. The results in Table 1 represent tensile strength in two perpendicular directions and tensile force per width for the films of $25 \mu \mathrm{m}$ thickness. The maximum force per width (and tensile strength) in the machining direction is about half of that for the transverse direction; however, the yield stress in the transverse direction is lower than that in the machining direction. In the case of uniaxial orientation, molecular orientation in amorphous polymers may influence the tensile strength by a factor of 3.[6] The film is biaxially oriented as a consequence of drawing. The large difference proves the anisotropic properties of the film, but it is not clear whether all the layers are affected in the same manner. However, it is the LDPE that has a low tensile strength compared to that of PA 6 and of $\mathrm{EVOH}$; hence, its influence on the result is not big and the large difference originates from PA 6 and $\mathrm{EVOH}$.

Table 1 Tensile properties of the film in two perpendicular directions.

\begin{tabular}{|l|c|c|}
\hline Tensile stress direction & Machining & Transverse \\
\hline Tensile strength, $\mathrm{MPa}$ & $31.9 \pm 0.1$ & $71.2 \pm 4.5$ \\
\hline Tensile force per width, N/mm & 0.80 & 1.78 \\
\hline
\end{tabular}

\subsection{Dynamic mechanical analysis (DMA)}

The dynamic mechanical properties are tested by means of the tensile dynamic mechanical analysis. In the analysis, molecules are mechanically excited and one can observe different relaxation processes in all the materials included in the multilayer film.

Due to the anisotropic behaviour, dynamic mechanical tests were performed in both the machining and the transverse direction. Only one surface of all the samples was directly exposed to radiation, either the LDPE or the PA 6, although it is quite certain that the UV light coming from the xenon-arc light source cannot be completely blocked by any layer of such a thin film (only $25 \mu \mathrm{m}$ thick). The layers can only reduce the intensity by partial absorption and reflection and this might influence the kinetics of induced reactions. In Figure 1 one can see how the mechanical spectra of both directions (i.e. machining and transverse) and both exposed surfaces (either PA 6 or LDPE) are affected by radiation for different exposure times. Figures 2 and 3 show the spectra with respect to the strain direction in the cases when either the PA 6 surface or the LDPE surface was directly irradiated, respectively.

The mechanically excited complex multilayer film undergoes a great number of relaxation processes in the large temperature range of almost $300 \mathrm{~K}$. In the application 
outdoors, the film will most probably be exposed to the temperature range between 275 and $325 \mathrm{~K}$. Luckily, it is obvious that radiation has the smallest influence on the mechanical damping, i.e. $\tan \delta$, exactly in this temperature range.

At low temperatures from 83 to $175 \mathrm{~K}$, several relaxation processes take place. PA 6 has a $\gamma$ - relaxation with the peak at $138 \mathrm{~K}$. The $\gamma$ - relaxation is caused by the segmental motion in the amorphous region with a small number of methylene groups between the amide groups.[7] Since the samples were not dried prior to the DMA, there is a so-called water peak for PA 6 that appears at approximately $143 \mathrm{~K}$ at $60 \%$ relative humidity (laboratory conditions), but its position may shift to higher temperatures with a large increase in the relative humidity. The same multilayer film was analysed after 28 days of being conditioned in water. The water-saturated film showed a rather pronounced water peak at $210 \mathrm{~K}$ coupled with the shift of the $\alpha$ - relaxation peak to lower temperatures.[8] Both effects are typical for PA 6.[3] At a temperature of $147 \mathrm{~K}$, the Schatzki relaxation has its maximum. This mechanism has been observed in many polymers containing linear $\left(\mathrm{CH}_{2}\right)$ sequences with $\mathrm{n}=4$ or greater and it is typical of polyethylene.[9,10] Isotropic EVOH does not have any relaxation process in this temperature range; however, according to [11], there is a $\gamma$ relaxation at $148 \mathrm{~K}$ for EVOH after drawing. This relaxation is visible in both the machining and the transverse direction.

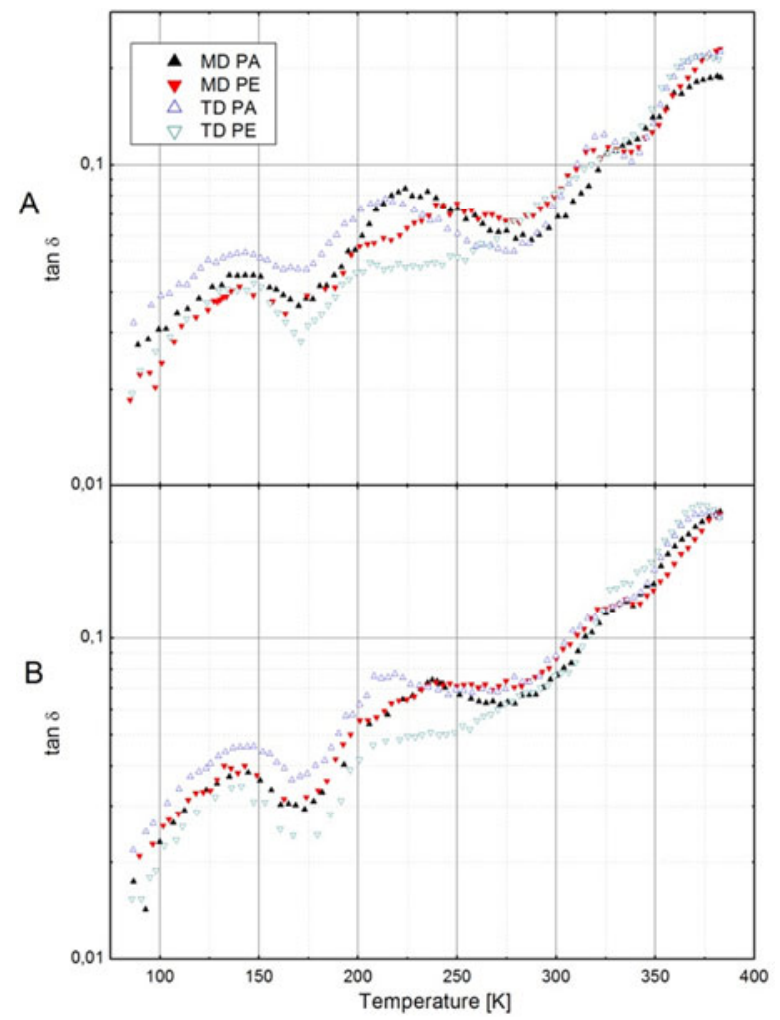

Fig. 1 Comparison of loss factor $(\tan \delta)$ of films when either the LDPE or the PA 6 surface was irradiated in both the machining and the transverse direction, after 3 hours (A) and 8 hours (B).

Irradiation does not affect the position of the peak in the low temperature range. The relaxation processes take place mainly due to segmental motions. Regardless of the reaction to radiation, there are still segments of comparable sizes and surroundings with comparable relaxation times in all layers; hence, the peak position remains the same. The position is independent of the radiation exposure time, of machining direction or the surface directly exposed to radiation. However, there are changes in the peak height. This is due to the total number of segments participating in the relaxation process. With the exception of the peak of the films stressed in the machining direction after PE was irradiated, in the low temperature 
range, $\tan \delta$ decreases with exposure time (Figures 2 and 3). There are changes in the spectra in both stress directions when the PA surface was exposed but they are similar; hence the relaxation mechanisms are not affected by the stress direction, but only by irradiation. The stress direction is of greater importance when the PE surface was irradiated. The machining direction after the eight hour exposure remained unaffected, whereas $\tan \delta$ in the transverse direction decreased. This leads to the conclusion that molecular orientation resulting from processing influences the Schatzki relaxation process. Figure 1 clearly shows that the curves in the machining direction undergo a drop in $\tan \delta$ in the same manner, independent of the surface which was irradiated. Up to the room temperature, a difference is visible when the samples were stressed in the transverse direction. The four known relaxation processes take place at temperatures of $138,143,147$, and $148 \mathrm{~K}$ and they are all covered by the first peak. After the 67 hour irradiation of the PA surface, one can notice that the peak splits and two maxima can be distinguished. It is the water peak that is between these two maxima (at 143 $\mathrm{K})$. The joint relaxation peak does not broaden; hence, the water peak does not shift, but vanishes. This leads to the conclusion that solubility of water molecules in the film decreases after being exposed to UV for a longer period of time.

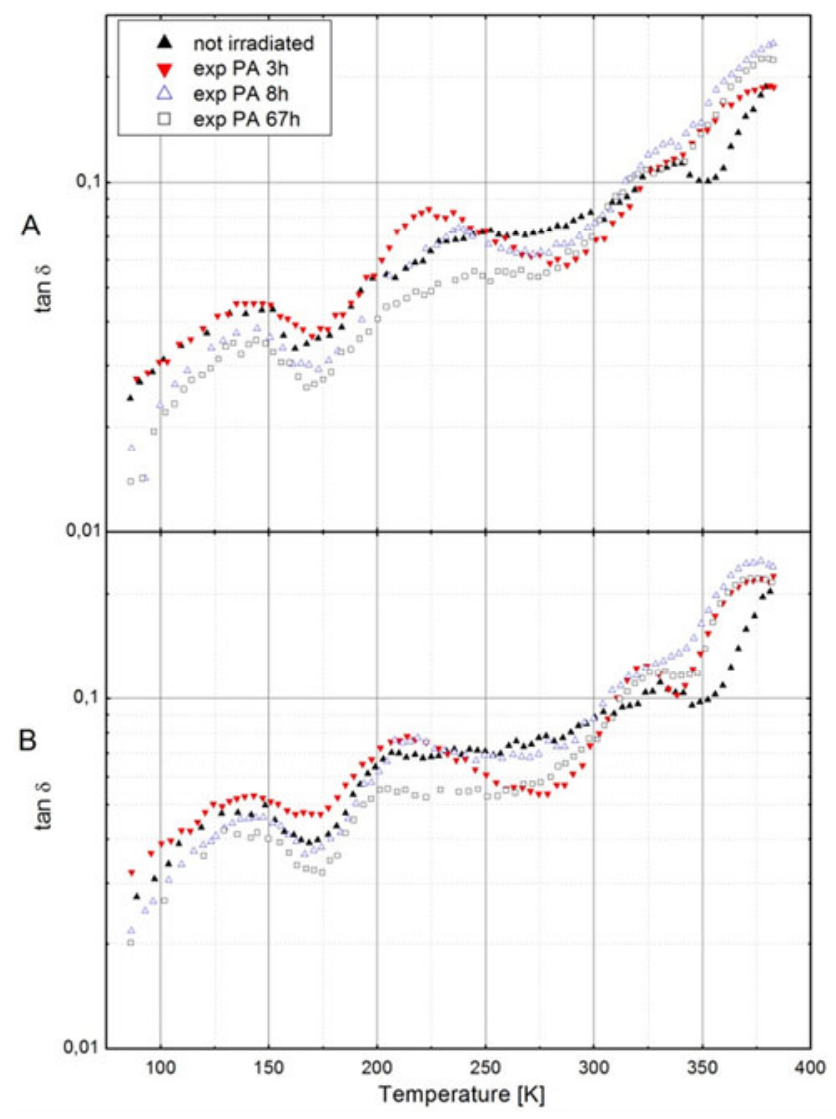

Fig. 2 Temperature dependence of loss factor $(\tan \delta)$ in the machining $(A)$ and the transverse direction (B) in the case when the PA 6 surface was exposed to xenon-arc light for different periods of time

The temperature range between 175 and $275 \mathrm{~K}$ includes several relaxation processes as well. At $208 \mathrm{~K}$, the $\beta$ - relaxation of PA 6 has its maximum. This relaxation is assigned to the motion of non-bonded amide groups in the amorphous region.[7] At a temperature of $253 \mathrm{~K}$, LDPE undergoes the $\beta$ - relaxation assigned to the amorphous regions of this semi-crystalline polymer or to the contribution of the side chains.[9] In their study, Kline et al.[12] also suggested that the $\beta$ - relaxation was associated with the relaxation of side groups or a shortchain branch. Although it was reported that EVOH has its $\beta$ - relaxation peak at the same temperature [13], measurements that were performed on EVOH monolayer films showed that 
this peak appears already at $230 \mathrm{~K}$, regardless y of the content of vinyl alcohol [3]. The $\beta-$ relaxation is ascribed to large-scale molecular motions of non-hydrogen-bonded chain sequences [13]. Measurements performed on the EVOH monolayer film with a different $\mathrm{PE}$ content under the same conditions showed that the film with a larger amount of PE (that is not hydrogen-bonded) has a more pronounced peak at $230 \mathrm{~K}$. According to the available literature, the three above mentioned processes are attributed to the relaxation of larger chain segments and side groups in the amorphous phase. Irradiation of the PE surface of films stressed in the machining direction did not influence this region. However, when the film was stressed in the transverse direction, the effect of weathering could be noted after only 3 hours of exposure and the prolonged exposure did not contribute to any further change. The value of tan $\delta$ exhibited a significant drop. This was not the case when the PA surface was exposed. When the PA surface was irradiated, a drop in $\tan \delta$ in both directions occurred only after a longer time of exposure. The late response in the case when PA was directly irradiated leads to the conclusion that it is the $\beta$ - relaxation of LDPE that is affected. However, here again, the effect is strongly dependent on the stress direction in the same manner as it was at lower temperatures (i.e. the curves for the machining direction undergo a drop in tan $\delta$ regardless of the surface which was irradiated and a difference is visible when samples were stressed in the transverse direction).

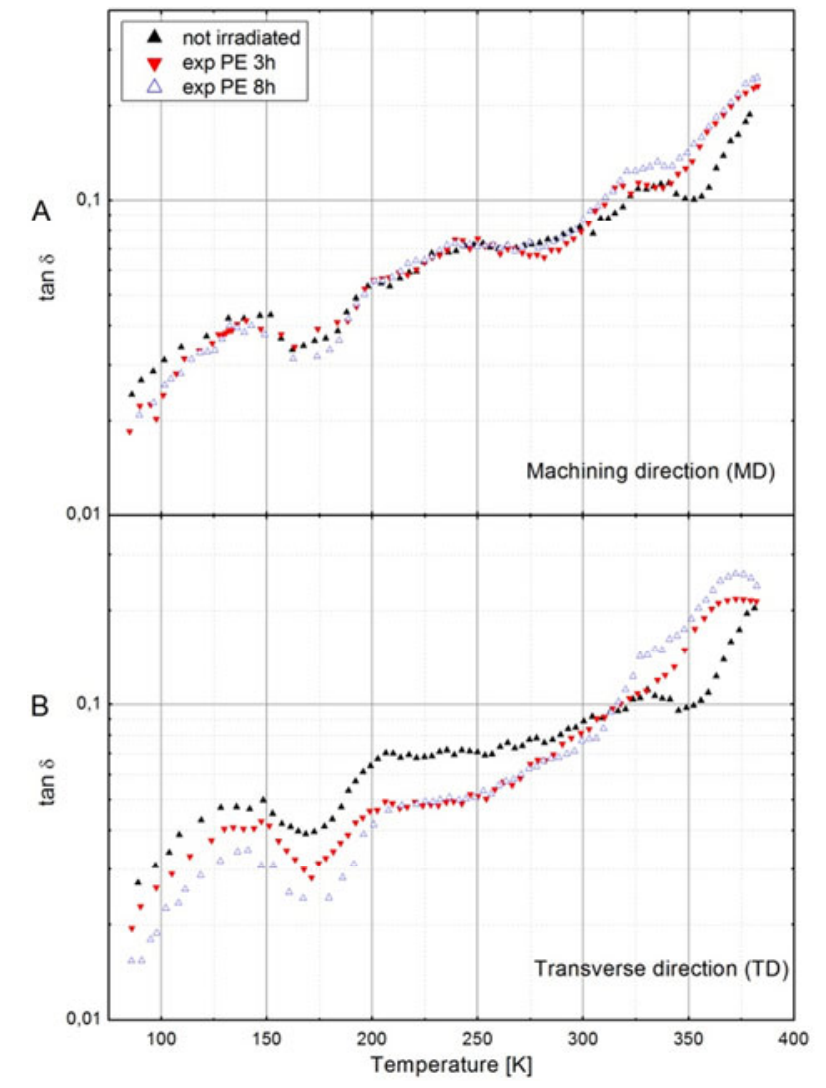

Fig. 3 Temperature dependence of loss factor $(\tan \delta)$ for the machining $(A)$ and the transverse direction (B) in the case when the LDPE surface was exposed to xenon-arc light for different periods of time

At temperatures above $293 \mathrm{~K}$, all three materials in the film go through relaxation processes as well. However, an overall trend in changes caused by irradiation is different from that at lower temperatures. 


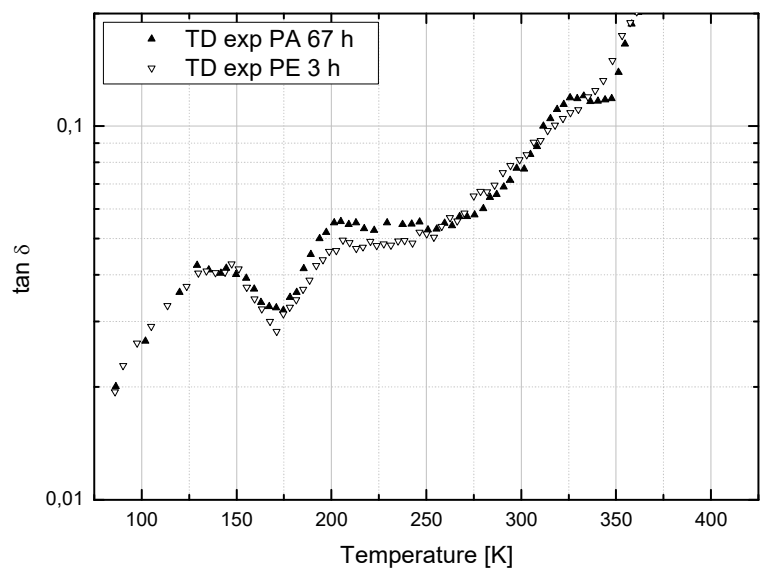

Fig. 4 Temperature dependence of loss factor $(\tan \delta$ ) for the transverse direction. The LDPE surface was irradiated for $3 \mathrm{~h}$ and the PA 6 for $67 \mathrm{~h}$. There is a large region of overlapping.

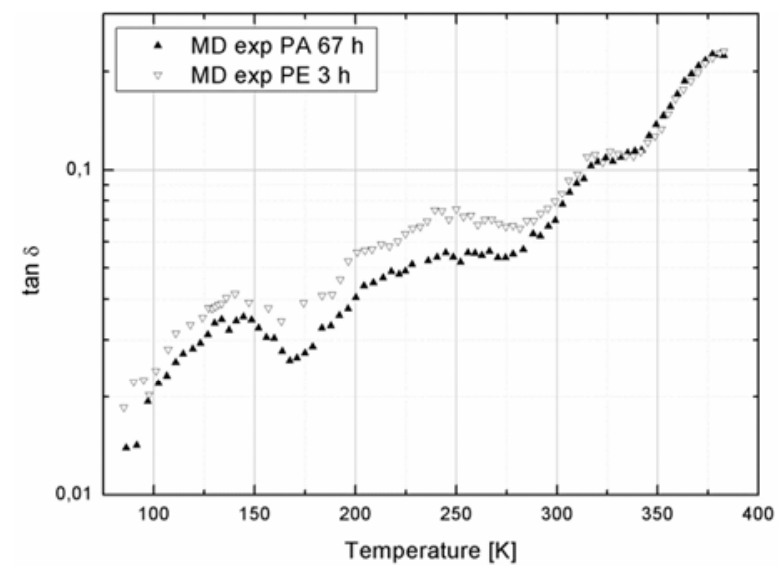

Fig. 5 Temperature dependence of loss factor $(\tan \delta)$ for the machining direction. The LDPE surface was irradiated for 3 hours and the PA 6 for 67 hours. The overlapping occurs only above $300 \mathrm{~K}$.

At a temperature of $325 \mathrm{~K}$, there is a peak for polyethylene that is attributed to its crystalline phase.[9] At $333 \mathrm{~K}$, the long-chain segmental motion in the amorphous region of PA 6 takes place as a result of rupture of hydrogen bonds between the polymer chains and this is the glass transition of PA 6.[7] Measurements performed on the EVOH monolayer with low moisture content (i.e. laboratory conditions) showed the glass transition peak at $343 \mathrm{~K}$. The EVOH monolayer has an additional peak at approximately $373 \mathrm{~K}$, which is the $\alpha$ ' - relaxation attributed to molecular motions in the crystalline phase.[4,13,14] At temperatures between $300 \mathrm{~K}$ and $350 \mathrm{~K}$, the glass transitions of both PA 6 and EVOH take place and there is a relaxation process related to the PE crystalline phase. The maximum of the joint peak for the tested multilayer film is at $333 \mathrm{~K}$. This is due to the fact that there are two layers of PA 6; hence, the relaxation peak attributed to its glass transition temperature is more pronounced than that of EVOH.

At the temperature range $300 \mathrm{~K}-350 \mathrm{~K}$, one can notice both an increase in the value of $\tan \delta$ and a shift to lower temperatures of both peaks ( $333 \mathrm{~K}$ and $373 \mathrm{~K}$ ). These effects are influenced by a higher mobility in the near- $T_{g}$ region. However, between 300 and $350 \mathrm{~K}$, the PE surface is more affected by the temperature than the PA surface (Figures 2 and 3). Since $\mathrm{PE}$ does not undergo glass transition in this temperature range, it is not only the $T_{g}$ that is influenced by irradiation, but also the crystalline phase in PE. The crystalline phase in EVOH is strongly affected as well. The respective peak at $373 \mathrm{~K}$ undergoes the largest shift regardless of the direction of irradiation or the surface that was irradiated (Figures 2 and 3). The increase in the height of the peak attributed to the crystalline phase of stretched EVOH indicates that there was an increase in the crystallinity degree of EVOH as well, but the shift 
to lower temperatures means that the activation energy for this relaxation process decreases. This might be caused by defects in the newly established crystals. From the intensity of the shift (Figure 1) it is obvious that the relaxation in the transverse direction takes place at slightly lower temperatures. The probable reason for that is that the crystalline phase in EVOH, a copolymer of ethylene and vinyl alcohol, has a preferential orientation [15] and its mobility is higher when the material is stressed in the transverse direction than in the machining direction. Although the stiffness of the film at high temperatures is too low for the majority of applications, the information on higher sensitivity of the crystalline phase of $\mathrm{EVOH}$ to the stress direction gives an insight into the change that is important for this material that is often used as a gas barrier.

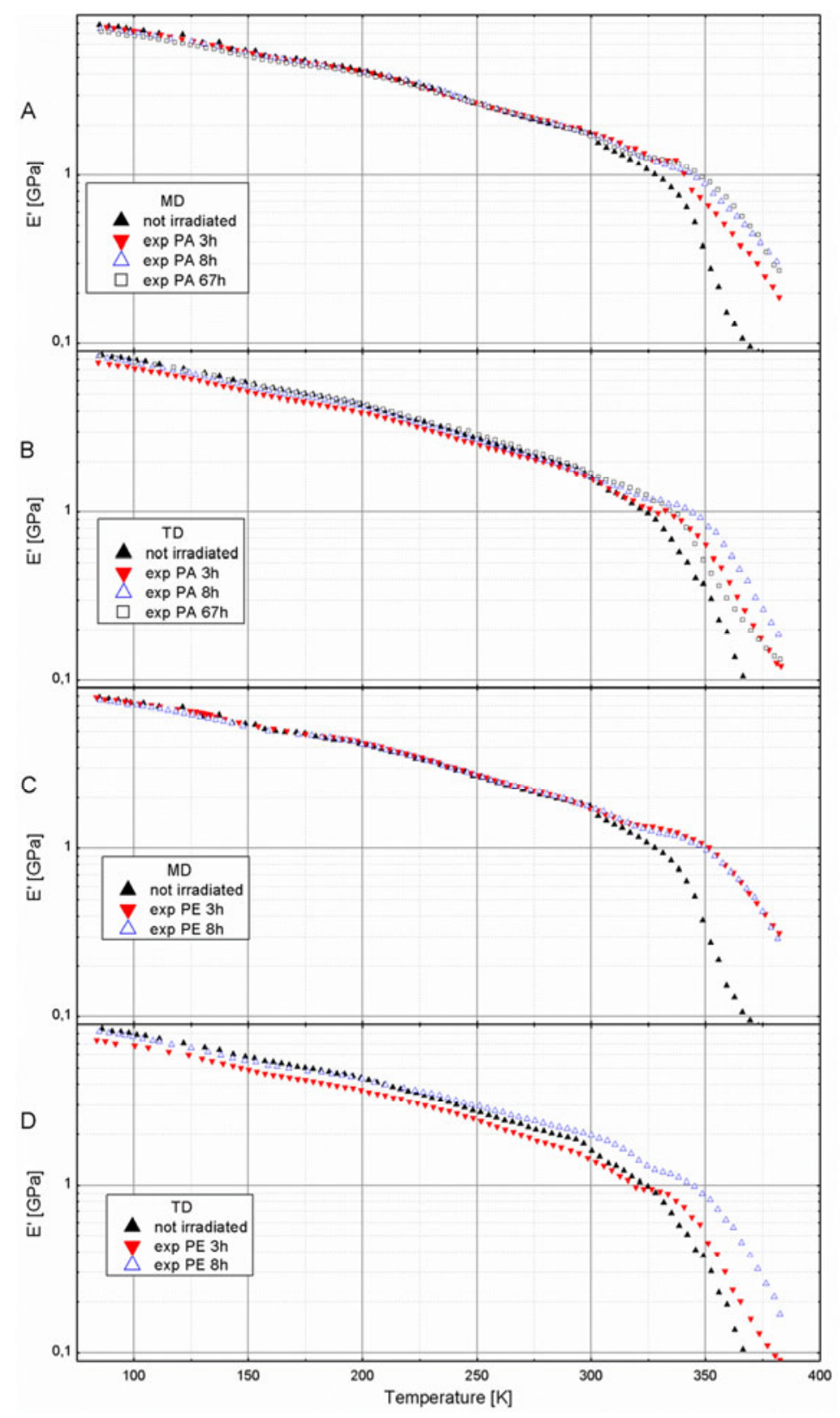

Fig. 6 Storage modulus for the machining direction (MD) and the transverse direction (TD) in the cases when either the PA 6 (A and B) or the LDPE surface of the film was directly exposed

A large overlap of $\tan \delta$ was noticed in two films, when the PA surface was exposed for 67 hours and when the PE surface was exposed for 3 hours (Figures 4 and 5). In the machining direction, the similarity was observed in the whole temperature range, whereas in 
the transverse direction, excellent overlapping was observed in the whole range except for temperatures between 175 and $275 \mathrm{~K}$. Polyolefins are known for their sensitivity to photooxidation when chain scission and cross-linking take place and result in cracks. [4] The layers of PA 6 and EVOH delay their degradation which was accelerated in the weathering chamber. The main advantage of LDPE is in its excellent welding properties, but the EVOH layer should be on the inner side when the film is exposed to the outdoor conditions.

The storage modulus measured by the DMA (Figure 6) is comparable to that measured by the tensile test machine and it is of great importance for application. At low temperatures, the film is very stiff for a polymer and the storage modulus is between 6 and $7 \mathrm{GPa}$. The higher the temperature the lower is the value of the modulus (at room temperature, it is about $2.2 \mathrm{GPa}$ ). The slope is monotonous with larger kinks at temperatures above $300 \mathrm{~K}$ where relaxation peaks are observed in the $\tan \delta$ vs temperature graphs. These kinks mark the onset of the glass transition of EVOH and PA 6. Similar to the $\tan \delta$ results, the effect of radiation is modest in the temperature range between 275 and $325 \mathrm{~K}$ (the actual temperatures in use); however the modulus is rather stable at lower temperatures as well. As expected, near the glass transition, i.e. above the temperature of approximately $325 \mathrm{~K}$, the modulus curve undergoes a marked change in the slope. At temperatures above $343 \mathrm{~K}$, there is no glassy region anymore and all the layers are in the rubbery state. The value of modulus decreases by one order of magnitude compared to that at $83 \mathrm{~K}$; a temperature rise of only $50 \mathrm{~K}$ results in a further drop by an additional order of magnitude.

Exposure to the solar spectrum makes the film stiffer, which leads to an increase in the storage modulus. Although $\tan \delta$ (i.e. molecular mobility) at higher temperatures increased with the exposure time, the moduli of the exposed films start to decrease at higher temperatures. In addition, the drop is slower than that of the unexposed film. The increase in the storage modulus and a shift of the glass-to-rubber transition to higher temperatures indicate less flexible segments. The shift in the onset and in the milder slope is due to the fact that the crystallinity degree increased, leading to a less amorphous phase that undergoes the glass-to-rubber transition. The shift in the onset to higher temperatures was probably due to the cross-linking.

\subsection{Fourier transform infrared (FTIR) spectroscopy}

Information on chemical changes in the irradiated film was obtained by the FTIR analysis.

Figures 7 and 8 show the spectra of the films when the PE surface was analysed by the FTIR spectroscopy. In Figure 7 it was the PE surface that was directly irradiated, while in Figure 8 it was the PA side.

The FTIR spectra of PE show characteristic bands of C-H stretching (asymmetric and symmetric) at 2916 and $2848 \mathrm{~cm}^{-1}$, bending deformation at 1462 and $1473 \mathrm{~cm}^{-1}$, and rocking deformation at 719 and $730 \mathrm{~cm}^{-1}$. Presence of the band at $1366 \mathrm{~cm}^{-1}$ indicates a level of branching within the polymer [16], and branching is typical of LDPE.

The duration of the exposure has little influence on the spectra of PE. The intensity of C-H stretching bands decreases slightly, but since this is not accompanied by the appearance of bands of carbonyl $\left(\sim 1710 \mathrm{~cm}^{-1}\right)$ or vinyl groups $\left(1630,970 \mathrm{~cm}^{-1}\right)$, the decrease cannot be ascribed to the oxidation or depolymerization of PE. This decrease is also somewhat less marked on the unexposed surface of the film in comparison with the exposed side, but in general there is no difference between the spectra of the exposed and the unexposed side. The lack of a visible change in the FTIR spectra does not mean there is no degradation: the main chain scission and the resulting cross-linking cannot be identified by the IR spectroscopy.[17] 


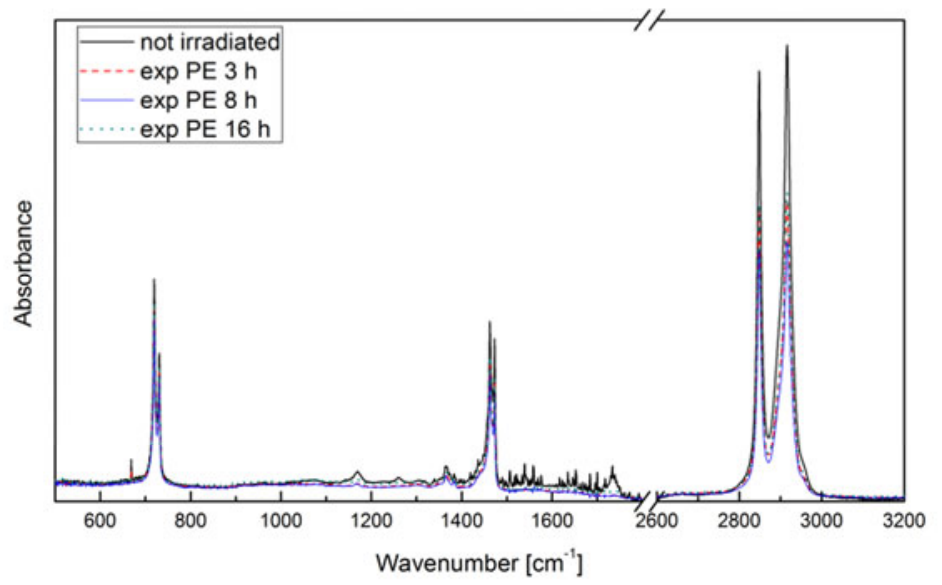

Fig. 7 FTIR spectra of the LDPE surface depending on the exposure time in the case when it was directly irradiated.

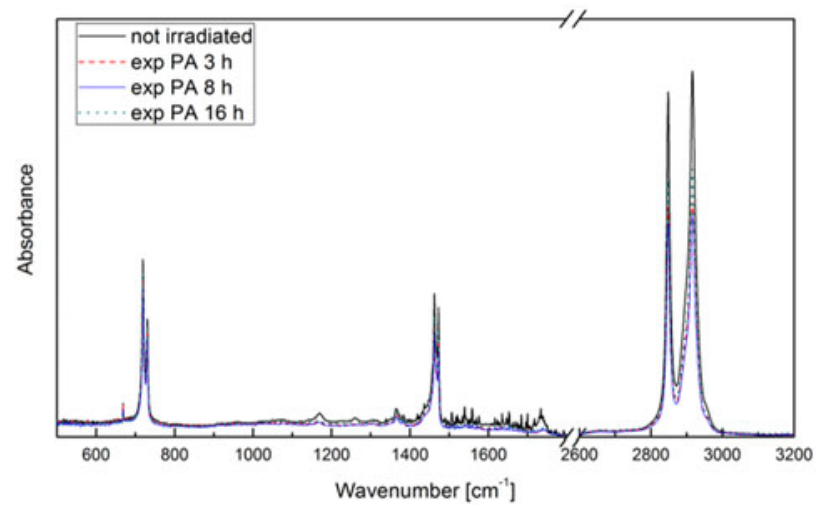

Fig. 8 FTIR spectra of the PE surface depending on the exposure time in the case when the PA surface was directly irradiated

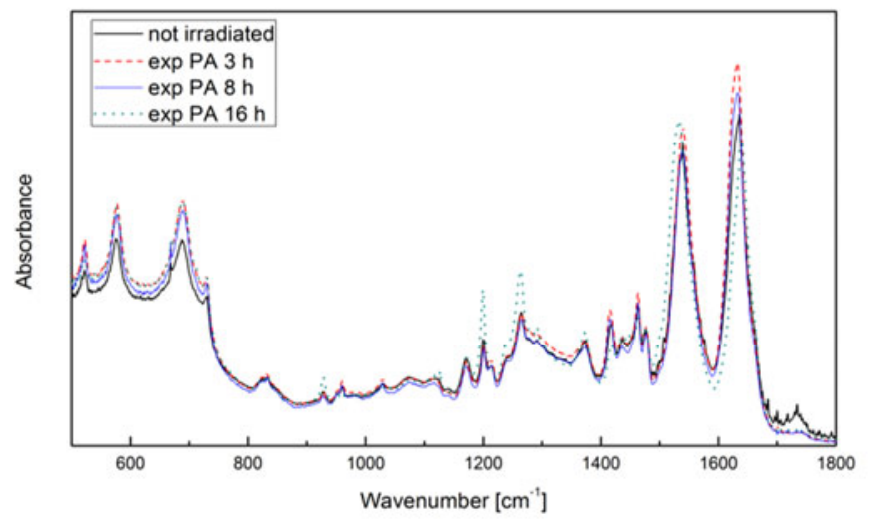

Fig. 9 The first part of the FTIR spectra of the PA surface depending on the exposure time in the case when it was directly irradiated 


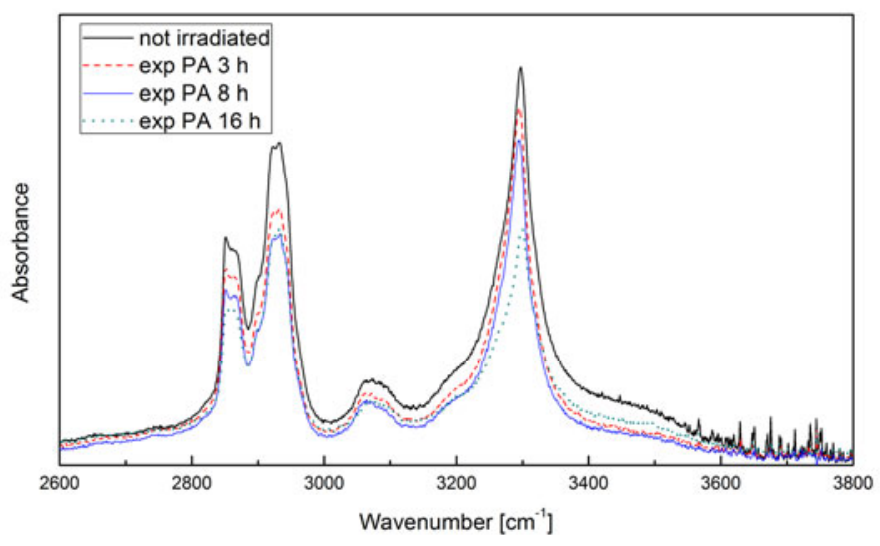

Fig. 10 The second part of the FTIR spectra of the PA surface depending on the exposure time in the case when it was directly irradiated

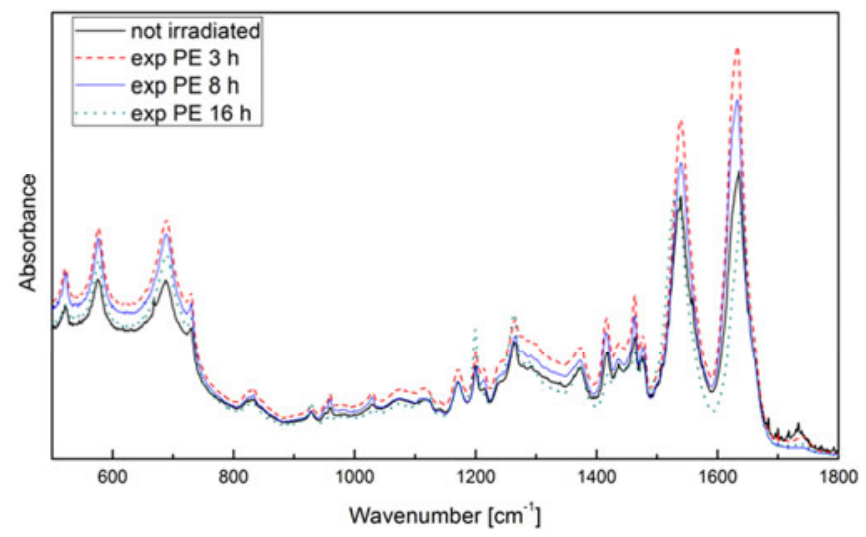

Fig. 11 The first part of the FTIR spectra of the PA surface depending on the exposure time in the case when the PE surface was directly irradiated

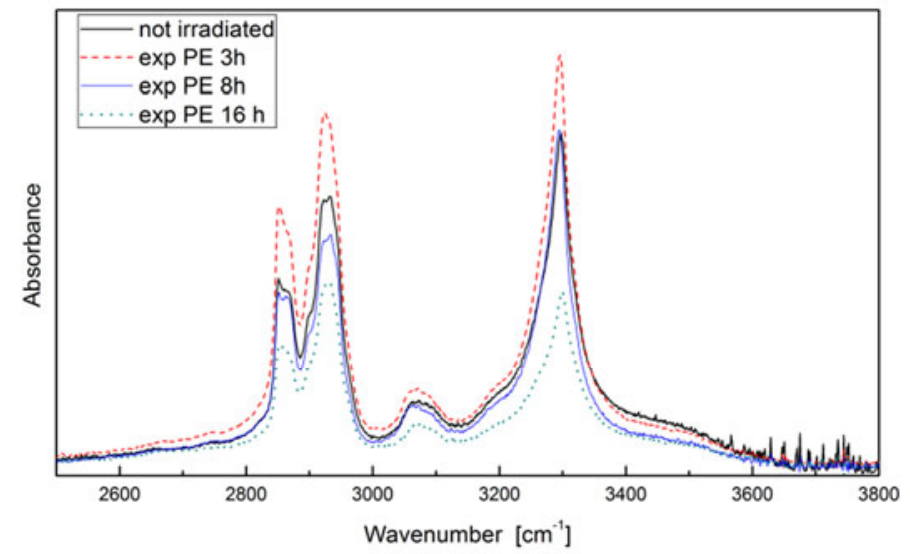

Fig 12 The second part of the FTIR spectra of the PA surface depending on the exposure time in the case when the PE surface was directly irradiated

When PA is concerned, there are two regions of interest that are shown in Figure 9 and Figure 10 in the case when the PA was the surface that was irradiated and in Figure 11 and Figure 12 when it was the PE surface that was irradiated. The FTIR spectra of polyamide show characteristic amide bands, as well as bands of $\mathrm{C}-\mathrm{H}$ stretching at 2930 and $2850 \mathrm{~cm}^{-1}$ and $\mathrm{N}-\mathrm{H}$ stretching of primary and secondary amines at $3297 \mathrm{~cm}^{-1}$. Amide bands are: $\mathrm{C}=\mathrm{O}$ stretching (amide I, $1632 \mathrm{~cm}^{-1}$ ), N-H in-plane bending (amide II, $1540 \mathrm{~cm}^{-1}$ ), the mixture of $\mathrm{C}-\mathrm{N}$ stretching and in plane N-H deformation (amide III, 1372 and $1263 \mathrm{~cm}^{-1}$ ), C-C=O 
stretching (amide IV, 958-952 $\mathrm{cm}^{-1}$ ) and N-H out-of-plane bending (amide V, $690 \mathrm{~cm}^{-1}$ ). The amide $\mathrm{B}$ (amide II overtone) band, due to the stretching of $-\mathrm{C}-\mathrm{N}$ groups, can also be observed at $\sim 3065 \mathrm{~cm}^{-1}$.[18-20]

Unlike PE, PA shows a considerable change in the spectra after weathering. There is a significant decrease in the C-H stretching bands at 2930 and $2850 \mathrm{~cm}^{-1}$, while the N-H band decreases and shifts to $3300 \mathrm{~cm}^{-1}$. Amide I and II bands shift from 1632 to 1639 and from 1540 to $1534 \mathrm{~cm}^{-1}$, respectively, which indicates decreased hydrogen bonding.[21] The band at $1730 \mathrm{~cm}^{-1}$, which can be ascribed to the C-O stretching in esters,[22] disappears with exposure. The disappearance is completed already after 3 hours on the exposed side, but the band is still present on the unexposed polyamide surface (Figure 13). A similar effect is also visible in the decrease in the $\mathrm{C}-\mathrm{H}$ and amide I bands, which is more pronounced on the exposed surface after 3 hours, but after 16 hours of exposure, the difference between the exposed and unexposed sides disappears.

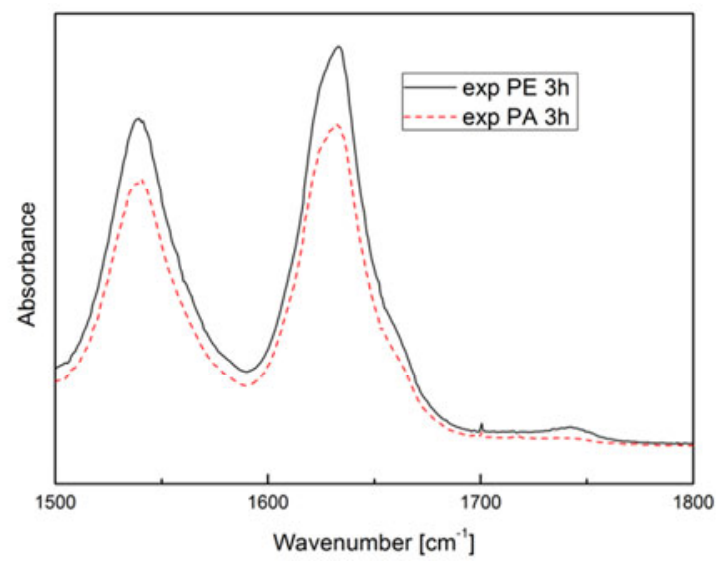

Fig. 13 Comparison of spectra after 3 hour exposure in the case when either the LDPE or the PA 6 surface was irradiated for 3 hours.

Long exposure also leads to considerable differences in the fingerprint region. Bands at $928,1125,1200$, and $1263 \mathrm{~cm}^{-1}$ increase, while those at 960,1030,1140, and $1215 \mathrm{~cm}^{-1}$ decrease. This indicates a change (an increase) in the degree of crystallinity.[17,19,20,23] The disappearance of the water peak in the DMA spectrum indicates that the solubility of water molecules in PA 6 decreased. These two results are in agreement since it is the amorphous phase that has a larger amount of free volume available for water molecules. In the area of C$\mathrm{H}$ deformation vibrations, the bands of $\mathrm{CH}_{2}$ in-plane bending at 1463 and $1418 \mathrm{~cm}^{-1}$ (attributed to the scissoring vibration of the $\mathrm{CH}_{2}$ unit next to the $\mathrm{NH}$ and to $\mathrm{CO}$ groups, respectively)[19,20] also decrease, while the bands at 1372 and $1450 \mathrm{~cm}^{-1}$ increase.

\subsection{Water vapour sorption}

Water vapour sorption was performed since the DMA measurements were not performed in a vacuum and the samples contained a certain amount of vapour.

It is well known that EVOH and PA will absorb vapour. Their properties are affected by the amount of free volume that is influenced by the vapour content.[4,24] Due to absorption, the mass of saturated PA 6 increases by $10 \%$,[3] whereas the water vapour absorbance of EVOH is influenced by the ethylene content. From the melting temperature range it is clear that the tested EVOH has low ethylene content. Since PE is non-polar and hydrogen bonds are established between the hydroxyl groups present in the vinyl alcohol fraction, the higher PE content would decrease the vapour absorbance of vinyl alcohol.[4] The absorbance of polyethylene itself should not be an issue because even if it absorbs some water vapour, it is the existence of hydrogen bonds between a polymer and a water vapour molecule that causes changes in properties.[3] 
TGA

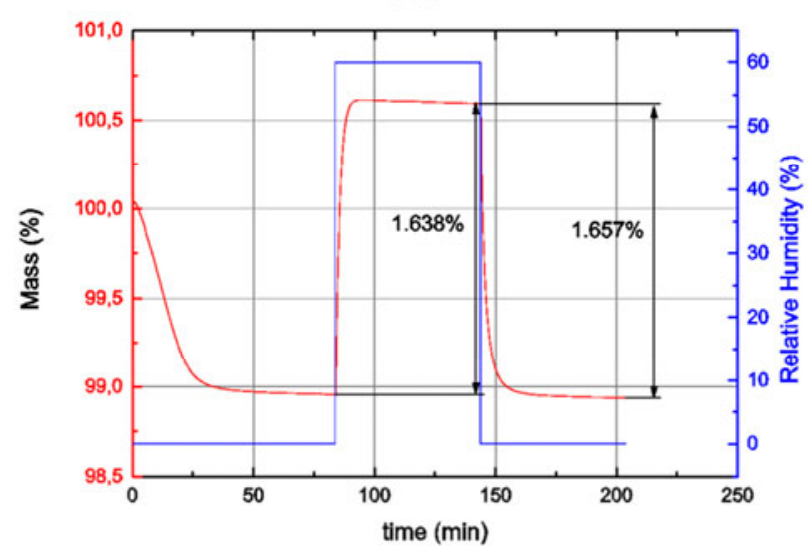

Fig. 14 The water vapour sorption test performed by TGA

The cycle is shown in Figure 14. It has three steps: drying, absorption, and desorption. The measurement shows that the mass fraction of the absorbed water vapour at $60{ }^{\circ} \mathrm{C}$ and 60 $\%$ relative humidity is in the $1.38 \pm 0.03$ mass $\%$. The mass after drying is $1 \mu \mathrm{g}$ or 0.017 mass $\%$ higher than after desorption. It is probably due to the fact that during the first 35 minutes of drying the temperature was below $60{ }^{\circ} \mathrm{C}$; this might be related to a slight swelling during absorption. Prior to the measurement, the film was stored at $20{ }^{\circ} \mathrm{C}$ and $60 \%$ relative humidity (laboratory conditions). From the drying cycle it is clear that in these conditions the film will absorb approximately $1.04 \pm 0.03$ mass $\%$.

From the drying cycle it is obvious that at room temperature the mass fraction of water vapour in the sample is less than $1.1 \%$.

\subsection{Melting temperature range}

The first range observed was 383 to $388 \mathrm{~K}$, the second was 479 to $488 \mathrm{~K}$, and the third was 495 to $500 \mathrm{~K}$. According to reference [25], LDPE has the melting temperature range from 378 to $388 \mathrm{~K}$, while the PA 6 has the melting point at about $496 \mathrm{~K}$,[6]; however, EVOH usually has lower melting temperature than the temperatures observed here. The high temperature indicates a low PE content in EVOH.

\section{Conclusion}

Mechanical properties of polymers are strongly influenced by temperature changes. The change in mechanical properties is by no means monotonous. The coextruded film exhibits large anisotropy, with an advantage of the transverse direction. The tested multilayer film when used outdoors should be turned with the PA surface facing outwards. Although the standardized 1000 hour weathering in a chamber with an exchange of „sunny“ (UV exposed) and ,rainy“ (demineralized water) cycles resulted in the disintegration of the tested film, the exposure of 67 hours only to UV light did not cause any dramatic visible change. This is due to the fact that PA is more sensitive to UV-induced oxidation in combination with rainy cycles [4] and the stability of PA in outdoor use is highly dependent on the number of rainy days. Maintenance costs are strongly influenced by changes in properties during the outdoor use. The selection of the film for a particular purpose should be governed by the number of sunny days, the irradiation intensity, and humidity.

The measured results of $\tan \delta$ and storage modulus $E$ ' in dry conditions prove that there is a certain change in mechanical properties in the actual temperature range in use (approximately from 250 to $310 \mathrm{~K}$ ). However, $\tan \delta$ around $273 \mathrm{~K}$ is hardly affected by irradiation and this is an advantage for the prediction of energy losses. At temperatures between 250 and $310 \mathrm{~K}$, the modulus $E$ ' decreases approximately by $50 \%$. However, after 
long-term irradiation (of about 67 hours), the film becomes stiffer. From the DMA and FTIR spectra it is clear that the degrees of crystallinity of PA 6 and $\mathrm{EVOH}$ increase, whereas the crystalline structure of low-density polyethylene (LDPE) is affected, but it is not clear whether it increases as well. The increase in the degree of crystallinity leads to a decrease in water sorption.

The complexity of the multilayer film hinders a detailed study of molecular dynamics. For a more detailed analysis, Raman spectroscopy should be performed.

\section{Acknowledgement}

This study was performed with financial support of the Croatian Science Foundation's Installation Grant and the European Community's Seventh Framework Program FP7ENERGY-2010-FET under the acronym HAWE.

We thank Mario Mačković and Ex Agencija for giving us the opportunity to use their weathering chamber. We also thank Ole Hölck and Jörg Bauer from Fraunhofer IZM MMCB Institute for the measurement of water vapour sorption.

\section{REFERENCES}

[1] Yam, K. L. The Wiley Encyclopedia of Packaging Technology, John Wiley \& Sons, Inc., New York, 2009

[2] Ehrenstein, G.; Pongratz, S. Resistance and Stability of Polymers (Vol. 1), Carl Hanser Verlag, Munich, 2013

[3] Baschek, G.; Hartwig, G.; Zahradnik, F. Effect of water absorption in polymers at low and high temperatures, Polymer 1999, 40, 3433-3441. https://doi.org/10.1016/s0032-3861(98)00560-6

[4] Cabedo, L.; Lagarón, J. M.; Cava, D. D.; Saura, J. J.; Giménez, E. The effect of ethylene content on the interaction between ethylene-vinyl alcohol copolymers and water (II): Influence of water sorption on the mechanical properties of EVOH copolymers, Polymer Testing 2006, 25 (7), 860-867.

https://doi.org/10.1016/j.polymertesting.2006.04.012

[5] Chen, H.; Ginzburg, V. V.; Yang, J.; Yang, Y.; Liu, W.; Huang, Y.; Du, L.; Chen, B. Thermal Conductivity of Polymer-Based Composites: Fundamentals and Applications, Progress in Polymer Science 2016, 59, 41-85. https://doi.org/10.1016/j.progpolymsci.2016.03.001

[6] van Krevelen, D. W.; te Nijenhuis, K. Properties of Polymers: Their Correlation with Chemical Structure, Elsevier Publishing Company, Amsterdam, 2009

[7] Ishisaka, A.; Kawagoe, M. Examination of the time-water content superposition on the dynamic viscoelasticity of moistened polyamide 6 and epoxy, Journal of Applied Polymer Science 2004, 93, 560567. https://doi.org/10.1002/app.20465

[8] Pavković, M. Effect of water and UV radiation on dynamic-mechanical properties of biaxially oriented ethylene-vinyl alcohol copolymer, Diploma thesis, Faculty of Mechanical Engineering and Naval Architecture, Zagreb 2014

[9] Lee, H.; Cho, K.; Ahn, T.-K.; Chole, S.; Kim, I.-J.; Park, I.; Lee, B. H. Solid-state relaxations in linear low-density (1-octene comonomer), low-density, and high-density polyethylene blends, Journal of Polymer Science Part B: Polymer Physics 1997, 35, 1633-1642. https://doi.org/10.1002/(SICI)10990488(19970730)35:10<1633::AID-POLB15>3.0.CO;2-B

[10] Shaw, M. T.; MacKnight, W. J. Introduction to Polymer Viscoelasticity, Third Edition, Wiley, 2005

[11] Cerrada, M. L.; Benavente, R.; Pérez, E.; Pereña, J. M. The effect of tensile drawing on the structure and relaxation processes in vinyl alcohol-ethylene copolymers, Polymer 2001, 42 (7), 3127-3138. https://doi.org/10.1016/s0032-3861(00)00698-4

[12] Kline, D. E.; Sauer, J. A.; Woodward, A. E. Effect of branching on dynamic mechanical properties of polyethylene Journal of Polymer Science 1956, 22, 455-462. DOI:10.1002/pol.1956.1202210211

[13] Gaucher-Miri, V.; Jones, G. K.; Kass, R.; Hiltner, A.; Baer, E. Plastic deformations of EVA, EVOH and their Multilayers, Journal of Materials Science 2002, 37 (13), 2635-2644. https://doi.org/10.1023/A:1015872129472

[14] Amini, M.; Mobli, M.; Khalili, M.; Ebadi-Dehaghani, H. Assessment of Compatibility in Polypropylene/ Poly(lactic acid)/Ethylene Vinyl Alcohol Ternary Blends: Relating Experiments and Molecular Dynamics Simulation Results, Journal of Macromolecular Science, Part B Physics 2018, 57:4, 287-304, https://doi.org/10.1080/00222348.2018.1460153 
[15] Michell, R. M.; Müller, A. J. Confined crystallization of polymeric materials Progress in Polymer Science 2016, 54-55, 183-213 https://doi.org/10.1016/j.progpolymsci.2015.10.007

[16] Deslandes, A.; Ionescu, M.; Karatchevtseva, I.; Siegele, R.; Cohen, D. D. Oxidation of polyethylene implanted with low energy magnesium ions, Nuclear Instruments and Methods in Physics Research Section B: Beam Interactions with Materials and Atoms 2013, 307, 624-629. https://doi.org/10.1016/j.nimb.2012.12.103

[17] Burillo, G.; Adem, E.; Muñoz, E.; Vásquez, M. Electron beam irradiated polyamide-6 at different temperatures, Radiation Physics and Chemistry 2013, 84, 140-144 https://doi.org/10.1016/j.radphyschem.2012.06.029

[18] Chernev, B. S.; Eder, G. C. Side effects in the application of polyamide 6 barrier materials for fuel tanks, Journal of Applied Polymer Science 2013, 127, 230-236. https://doi.org/10.1002/app.37868

[19] Onder, E.; Sarier, N.; Sabri Ersoy, M. The manufacturing of polyamide- and polypropylene-organoclay nanocomposite filaments and their suitability for textile applications, Thermochimica Acta 2012, 543, 3758. https://doi.org/10.1016/j.tca.2012.05.002

[20] Porubská, M.; Szöllős, O.; Kóňová, A.; Janigová, I.; Jašková, M.; Jomová, K.; Chodák, I. FTIR spectroscopy study of polyamide- 6 irradiated by electron and proton beams, Polymer Degradation and Stability 2012, 97, 523-531. https://doi.org/10.1016/j.polymdegradstab.2012.01.017

[21] Dayma, N.; Jaggi, H. S.; Satapathy, B. K., Post-yield crack toughness behavior of polyamide6/polypropylene grafted maleic anhydride/nanoclay ternary nanocomposites, Materials \& Design 2013 , 49, 303-310. https://doi.org/10.1016/j.matdes.2013.01.011

[22] Tang, C. Y.; Kwon, Y.-N.; Leckie, J. O. Effect of membrane chemistry and coating layer on physiochemical properties of thin film composite polyamide RO and NF membranes: I. FTIR and XPS characterization of polyamide and coating layer chemistry, Desalination 2009, 242, 149-167. DOI:10.1016/j.desal.2008.04.003

[23] El-Mazry, C.; Correc, O.; Colin, X. A new kinetic model for predicting polyamide 6-6 hydrolysis and its mechanical embrittlement. Polym. Degrad. Stab. 2012, 97, 1049-1059. https://doi.org/10.1016/j.polymdegradstab.2012.03.003

[24] Zhang, Z.; Lim, L.-T.; Tung, M. A. Limonene Transport and Mechanical Properties of EVOH and Nylon 6,6 Films as Influenced by RH, Journal of Applied Polymer Science 2001, 79, 1949-1957. https://doi.org/10.1002/1097-4628(20010314)79:11<1949::AID-APP1002>3.0.CO;2-F

[25] Abdel-Bary, E. M. Handbook of Plastic Films, Rapra Technology Limited, Shawbury, Shrewsbury, Shropshire, 2003

$\begin{array}{lll}\text { Submitted: } & \text { 17.4.2019 } & \text { Tatjana Haramina } \\ & & \text { Lovro Fulanović } \\ \text { Accepted: } & 20.6 .2019 & \text { Faculty of Mechanical Engineering and } \\ & & \text { Naval Architecture, University of Zagreb, } \\ & \text { Zagreb, Croatia } \\ & \text { Jelena Macan } \\ & \text { Faculty of Chemical Engineering and } \\ & \text { Technology, University of Zagreb, } \\ & \text { Zagreb, Croatia }\end{array}$

